\title{
APLICACIÓN ACCESIBLE DE REPRODUCCIÓN DE MÚSICA VÍA STREAMING PARA ROBOT SOCIAL
}

\author{
M. A. Quispe-Flores, Sara Carrasco-Martínez, Javier Sevilla-Salcedo, Jaime Gómez-Jiménez \\ Fernando Alonso-Martín, Miguel A. Salichs \\ RoboticsLab, Departamento de Ingeniería de Sistemas y Automática, \\ Universidad Carlos III de Madrid \\ \{mquispe, sacarras, jasevill\}@pa.uc3m.es, 100383149@alumnos.uc3m.es, \{famartin, salichs\}@ing.uc3m.es
}

\section{Resumen}

La robótica social continúa adaptándose a los cambios que se están produciendo en el mundo y la sociedad a día de hoy. La aparición del COVID-19 ha supuesto un escenario de distanciamiento social que ha afectado en gran medida a poblaciones de riesgo como el de las personas mayores.

No obstante, las diversas intervenciones por parte de la robótica social, han demostrado su capacidad de combatir una pandemia, ofreciendo servicios en hospitales, hogares de ancianos y brindando compañia a personas mayores que viven solas. Dando como resultado la idea de impulsar el valor del robot social como plataforma de servicios.

Por este motivo, el presente trabajo propone el diseño e implementación de una aplicación de reproducción de música y podcasts enfocada en los gustos de las personas mayores. Nuestro desarrollo busca promover la accesibilidad y el uso de servicios de streaming de contenido multimedia en personas de edad avanzada. En paralelo, la aplicación desarrollada apunta a la creación de nuevas aplicaciones Android que apoyen a los robots sociales de la Universidad Carlos III de Madrid e incrementen sus competencias en materia de servicios.

Palabras clave: Robótica social; Servicios de streaming; Aplicaciones accesibles; Reproducción de música; Inclusión digital.

\section{INTRODUCCIÓN}

En la actualidad, el rápido desarrollo de la tecnología y la ciencia impulsa a la robótica en campos como la medicina, la industria o los servicios. Al mismo tiempo, la robótica social, rama más estrecha con la interacción humana, utiliza robots que se comunican mediante diversos canales como el uso de interfaces gráficas, gestos o diálogos. De cara a la interacción, estos recursos consiguen que el usuario sienta una sensación más cercana y amigable.

Sin embargo, el papel que desempeña la robótica social dentro del sector servicios todavía se encuentra en sus comienzos [4]. En estos últimos años, la robótica social se ha ido acercando a los hogares gracias a la continua integración de nuevas habilidades y aplicaciones con el objetivo de ser más atractivos hacia los nuevos usuarios.

Por otro lado, la situación actual del COVID-19 ha provocado que la sociedad enfrente un escenario perjudicial. Uno de las medidas tomadas para evitar su contagio fue el del aislamiento social, lo que supuso fuertes repercusiones en la salud a nivel mental [16], afectando en mayor medida a la población de las personas mayores, población de más alto riesgo. Con este antecedente, nace un nuevo enfoque de desarrollo de robots sociales centrado en servicios que apoyen a poblaciones de riesgo ante el escenario del COVID-19 [7].

Siguiendo esta línea, los robots sociales siguen adaptándose a los cambios que van surgiendo en el mundo y en la sociedad. Enfocándonos en las dificultades que encuentran las personas mayores a la hora de desenvolverse con la tecnología, nace la idea de desarrollar aplicaciones fáciles de utilizar y que ofrezcan contenido pensado para ellos. La accesibilidad de dichas aplicaciones se implementa en paralelo con el desarrollo de robots más accesibles de cara a la comunicación.

El trabajo que se presenta en este artículo consiste en el diseño e implementación de una aplicación de reproducción de música y podcast en el robot social Mini [19], robot creado por la Universidad Carlos III de Madrid con la finalidad de ayudar a personas con deficiencia neurodegenerativa de cara a realizar actividades de estimulación cognitiva [18]. La evolución de dicho robot ha permitido integrar aplicaciones como juegos, agendas personales o reproductores de contenido multimedia. Por lo tanto, se pretende desarrollar un servicio que brinde la oportunidad a las personas mayores de poder reproducir y tener a su disposición una amplia librería de su música favorita.

El resto del artículo se estructura de la siguiente manera: La sección 2 ofrece una revisión del estado del arte relacionado con la robótica social y las aplicaciones accesibles. La sección 3 ofrece las principales características que podría implementar la aplicación propuesta. La sección 4 presenta 


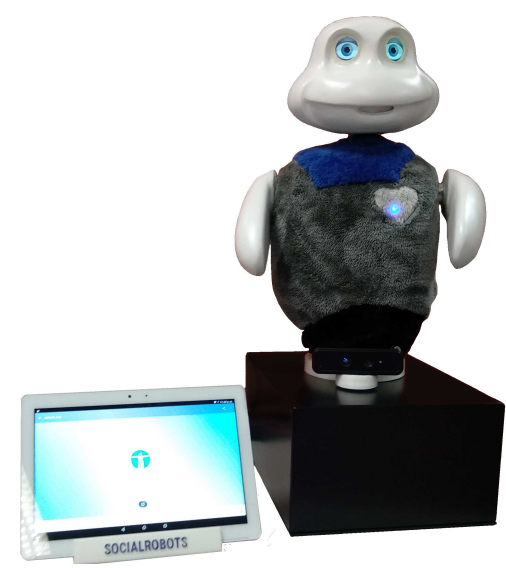

Figura 1: Robot social Mini [12]

la aplicación desarrollada, la cual incluye su diseño de manera más detallada. Por último en la sección 5 se presentan las conclusiones obtenidas tras la realización de este trabajo.

\section{ESTADO DEL ARTE}

La historia de la robótica nos demuestra que los robots que inicialmente fueron creados como productos de investigación han ido evolucionando al punto de convertirse en robots comerciales [15]. Estos han conseguido desenvolverse fuera del entorno del laboratorio al que principalmente fueron destinados, para poder integrarse en el entorno doméstico.

\subsection{ROBOTS CON REPRODUCTOR MULTIMEDIA}

Paralelo al desarrollo de los robots sociales, aparecen los altavoces inteligentes, capaces de ofrecer un rango más amplio de servicios y contenido multimedia. Además, cuentan con sofisticados sistemas ASR (Automatic Speech Recognition) y módulos de síntesis de voz. Analizando el mercado actual sobresalen los sistemas de diálogo como Cortana de Microsoft, Siri de Apple, Amazon Alexa o Google Assistant [8].

Por su parte, la robótica social ha conseguido integrar pantallas y altavoces inteligentes, creando autómatas capaz de proporcionar al usuario la capacidad de escuchar música, información de actualidad, pronóstico del tiempo o reproducción de juegos. Estas habilidades que inicialmente encontramos en altavoces inteligentes, han ido evolucionando a los robots sociales hasta el punto de ser considerados asistentes personales. Alguno de los ejemplos son robots como Pepper[14], SARA ${ }^{1}$ o Care-O-bot 4 [10], los cuales comparten carac-

\footnotetext{
${ }^{1}$ https://sara-robotics.com/
}

terísticas humanoides y disponen de una pantalla como interfaz gráfica. También existen robots de mesa o escritorio que integran una tablet o un dispositivo móvil. Es el caso de Jibo[6], ElliQ ${ }^{2}$ o $\mathrm{Mabu}^{3}$.

Finalmente, lo que se busca con estos nuevos diseños es abrir un abanico amplio de servicios para que el usuario pueda entretenerse y le resulte sencillo utilizar dichas aplicaciones.

\subsection{APLICACIONES PARA PERSONAS MAYORES}

En pleno siglo XXI, estamos viviendo un rápido incremento del envejecimiento de la población, en paralelo a un desarrollo veloz de las TIC (Tecnologías de la Información y la Comunicación) [5].

Abordando este último punto, existen sectores de población que no participa de las TIC, lo que da lugar a que se incremente una brecha digital entre generaciones. Una de las poblaciones más afectadas es el de las personas mayores.

Para minimizar dicha brecha se recurren a proyectos y programas que ayuden a mejorar su grado de manejo de dispositivos como teléfonos inteligentes y tabletas digitales [21]. Por otro lado, la creación de aplicaciones más intuitivas, accesibles y centrados en usuarios mayores ayuda en su conjunto a minimizar dicha brecha.

A continuación, se presenta un clasificación de aplicaciones desarrolladas para personas mayores [13], en relación al tema al que se dedican:

- Aplicaciones relacionadas con la salud y el ejercicio físico: como son las aplicaciones Podómetro, Medisafe ${ }^{4}$ y Medicamento Accesible Plus. Este último te permite consultar qué medicamentos debes tomar.

- Aplicaciones relacionadas con el ocio y el entretenimiento: como puede ser la aplicación Fit Brains Trainer, basada en juegos que entrenan la mente del usuario.

- Aplicaciones relacionadas con la accesibilidad: las cuales facilitan el manejo del dispositivo agrandando el teclado, los botones y la interfaz o transcribiendo la voz a la hora de enviar mensajes. Encontramos aplicaciones como Big Launcher ${ }^{5}$, Wiser y Dragon Anywhere.

\footnotetext{
${ }^{2}$ https://elliq.com

${ }^{3}$ http://www.cataliahealth.com/

${ }^{4}$ https : //www.medisafe.com

${ }^{5}$ http://biglauncher.com/es/
} 


\section{PROPUESTA}

La aplicación que se propone en este trabajo busca desarrollar un servicio de reproducción de música y podcasts vía streaming para el robot social Mini.

A la hora de realizar el diseño de la aplicación se deben tener en cuenta aspectos funcionales como la correcta ejecución y conexión al servicio vía streaming. Además, debe existir un correcto enlace entre la aplicación y el robot de cara al uso de sus módulos. Este último aspecto permite que la aplicación desarrollada utilice directamente los módulos ASR [2] y ETTS [3] (Emotional-Text-ToSpeech) del robot, mejorando la accesibilidad y experiencia del usuario.

De esta manera, se pretende iniciar una nueva línea de creación de nuevas aplicaciones que puedan ofrecer servicios como la reproducción de música, mensajería instantánea o cualquier recurso de internet, sin olvidar el aspecto de apoyo e integración digital de usuarios mayores.

Siguiendo las consideraciones precedentes, la aplicación propuesta está pensada para implementar un amplio catálogo de listas de música y programas de podcasts centrado en los gustos musicales de la población mayor. Ciertamente las preferencias musicales dependen de cada usuario, no obstante, nuestra propuesta pretende implementar una biblioteca que abarque numerosos géneros musicales basado en las preferencias más frecuentes de dichos usuarios.

El objetivo final persigue desarrollar al robot Mini como un robot social de servicios apoyado en múltiples aplicaciones más intuitivas, dinámicas y accesibles, aplicaciones que podríamos encontrar fácilmente en cualquier dispositivo móvil.

\subsection{CARACTERÍSTICAS GENERALES}

A continuación, se describen las características generales que debe presentar la aplicación propuesta.

\subsubsection{Sistema operativo}

La aplicación debe ser desarrollada en el lenguaje nativo de Android [11]. Dicho sistema se encuentra actualmente presente en la gran mayoría de dispositivos tabletas digitales y móviles.

Las herramientas de desarrollo oficiales, como Android Studio [1], nos permiten crear aplicaciones con diseños más personalizados. El soporte directo de Google nos permite utilizar distintos servicios y enfocar su implementación a una amplia gama de dispositivos. Además, las aplicaciones se basan en Java, un lenguaje global bastante sencillo de entender e implementar.

\subsubsection{Servicio streaming}

De cara a la elección del servicio de streaming se propone usar Spotify [9]. En la actualidad existen numerosas plataformas de streaming, las cuales son competencia directa de Spotify por la calidad de su contenido y número de usuarios [17].

Sin embargo, Spotify es el único servicio que además de liderar su mercado [20], cuenta con una gran comunidad y soporte para los desarrolladores de aplicaciones. Ofrece un SDK (Software Development Kit) para Android el cual se basa en un conjunto de librerías que proporcionan un control remoto sobre la propia aplicación de Spotify. De esta forma, se puede llegar a reproducir cualquier contenido guardado en su base de datos.

\subsubsection{Modos de conexión}

Otra de las características principales de la aplicación debe ser la capacidad de ofrecer al usuario dos tipos de uso basados en su conexión con el robot.

El sistema debe permitir los siguientes dos modos:

- Modo de conexión con el robot: permitiría a la aplicación hacer uso de los módulos ASR y ETTS del robot. De esta forma, el usuario podría controlar la aplicación mientras interactúa directamente con el robot, facilitando su accesibilidad y experiencia de uso. Sería el modo de conexión principal.

- Modo de conexión sin el robot: iniciaría la aplicación independiente al robot. Se trataría de un modo de conexión secundario o alternativo que permitiría al usuario, más familiarizado con la aplicación, controlarla libremente.

Sin embargo, ambos modos de conexión deben compartir el mismo sistema de menús y las mismas librerías de contenido.

\section{EJEMPLO PRÁCTICO}

En la actualidad, se ha desarrollado una aplicación que cumple con todas las características descritas en el apartado anterior. Esta aplicación se ha desarrollado principalmente como nuevo servicio para el robot social Mini.

Para el desarrollo de la aplicación se han usado las librerías del proyecto android-core, proyecto oficial del entorno Android-ROS ${ }^{6}$. El progra-

\footnotetext{
${ }^{6}$ http://wiki.ros.org/android
} 
ma se ha desarrollado bajo el subproyecto android_tutorial_pubsub, un programa simple que se conecta al entorno de ROS (Robot Operating System). Por lo tanto, la aplicación se ha desarrollado partiendo de este subproyecto, el cual cuenta con las funcionalidades básicas para implementar una correcta comunicación con los módulos del robot.

El sistema implementa el SDK de Spotify ${ }^{7}$, el cual permite conectarse remotamente a la aplicación de Spotify del dispositivo y controlarla. De esta forma, Spotify se encarga directamente de la reproducción y la conexión a la red. Mientras que nuestra aplicación se encarga de ofrecer al usuario una experiencia más accesible de reproducción del contenido.

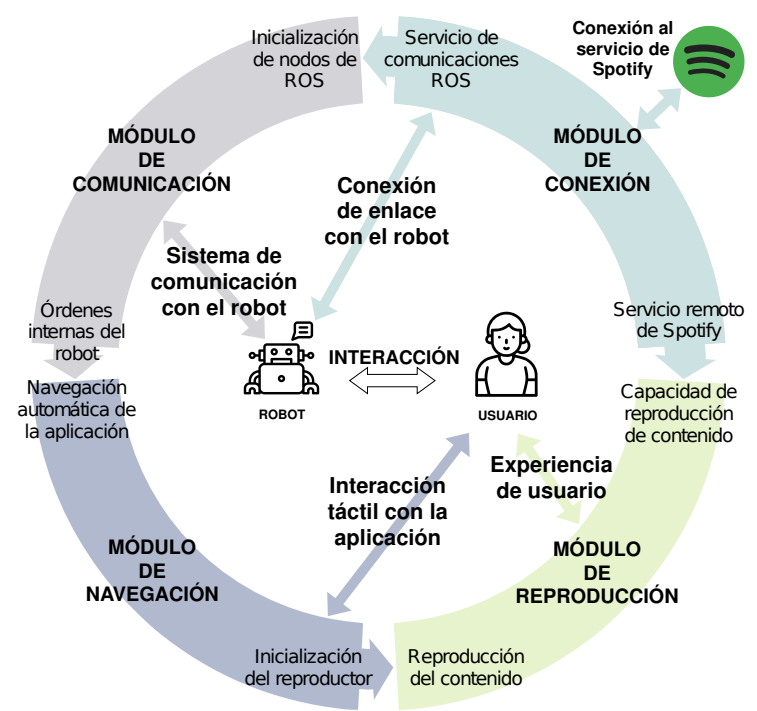

Figura 2: Esquema de los principales módulos de la aplicación y sus correspondientes relaciones

El diseño de la arquitectura de la aplicación se divide en cuatro partes principales: comunicación, conexión, navegación y reproducción de contenidos. La interrelación entre dichos módulos implementa la funcionalidad total del programa. Por lo tanto, cada parte es imprescindible de cara a desarrollar una correcta experiencia de usuario.

\subsection{ARQUITECTURA DEL SISTEMA}

La arquitectura de la aplicación se divide en cuatro módulos principales:

\subsubsection{Módulo de comunicación}

El módulo de comunicación permite al robot enviar mensajes con información relevante durante el ciclo de ejecución de la aplicación. Por lo tanto, permite a la aplicación utilizar el ASR y ETTS del robot, paralelo al control directo del robot sobre la

\footnotetext{
${ }^{7}$ https://developer.spotify.com
}

aplicación. Esto facilita que el usuario pueda manejar el programa con la mínima interacción sobre la pantalla de la tableta digital.

El resultado final permite que la aplicación se puede controlar mediante órdenes preestablecidas en los mensajes. La estructura del módulo (ver Fig. 3a) consta de dos partes principales: un conjunto de mensajes compuestos y una estructura de nodos de ROS.

\subsubsection{Módulo de conexión}

El módulo de conexiones permite a la aplicación establecer los servicios necesarios para el funcionamiento del módulo de comunicación y los servicios remotos de Spotify. Se trata del módulo más importante dentro del sistema, debido a que enlaza la aplicación al robot y le proporciona la capacidad de reproducir música sin limitaciones.

El módulo de conexiones está formado por una conjunto de etapas ordenadas según su ciclo de ejecución (ver diagrama de la Fig. 3b).

Las tres etapas principales que lo forman son:

- Etapa de Pre-selección: es la primera etapa del módulo y se inicia a la vez que la aplicación. En ella se configura e inician las actividades y servicios necesarios para la ejecución de la etapa de Selección.

- Etapa de Selección: tras la finalización de la etapa de Pre-selección, se muestra al usuario una interfaz gráfica con los dos posibles modos de conexión de la aplicación. Según el resultado de la elección, variarán en consecuencia el flujo de funcionamiento de la etapa y las conexiones a establecer.

- Etapa de Post-selección: última etapa del módulo donde se establecen las conexiones determinadas en la etapa anterior. Una vez las conexiones se hayan establecido correctamente, la etapa finaliza con la interfaz gráfica del menú principal.

\subsubsection{Módulo de navegación}

El módulo de navegación permite atender a las acciones por pantalla del usuario, y navegar entre los diferentes menús que componen la aplicación. Además se encarga de ejecutar las órdenes del robot, trabajando en conjunto con el módulo de comunicaciones.

Por otro lado, el programa sigue una estructura de menús ordenada por niveles, que permite al módulo moverse entre las diferentes opciones hasta llegar a los menús de selección. Éstos últimos inician el reproductor de música de la aplicación. 


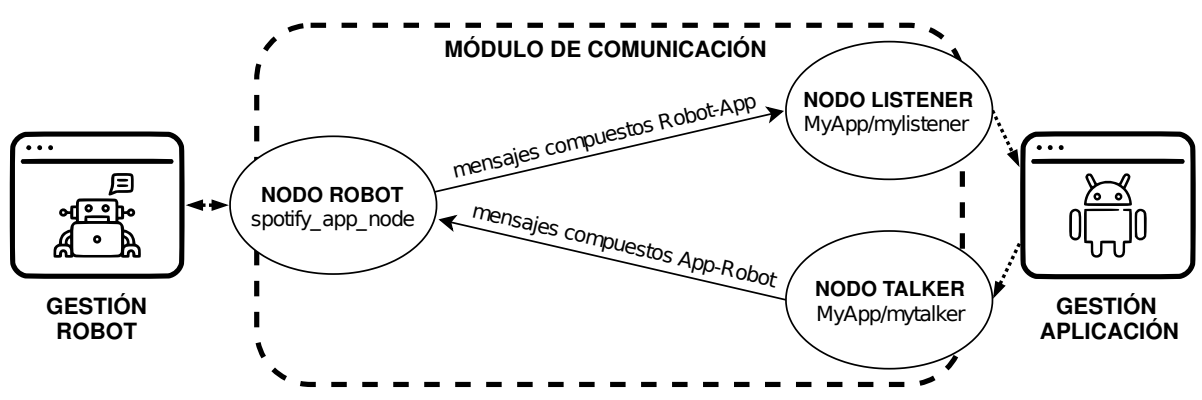

(a) Esquema de la estructura del módulo de comunicación

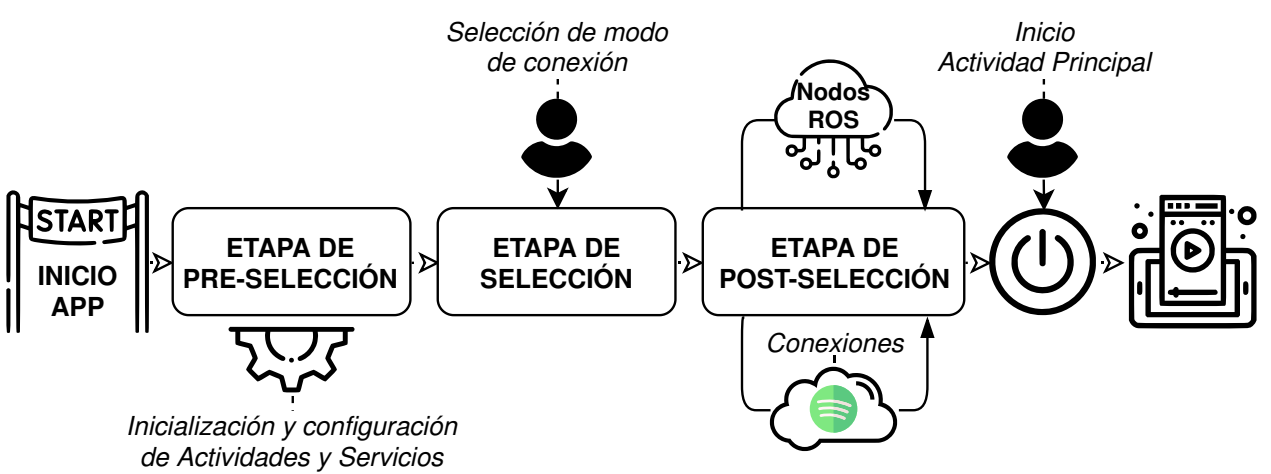

(b) Diagrama de secuencia de las etapas del módulo de conexiones

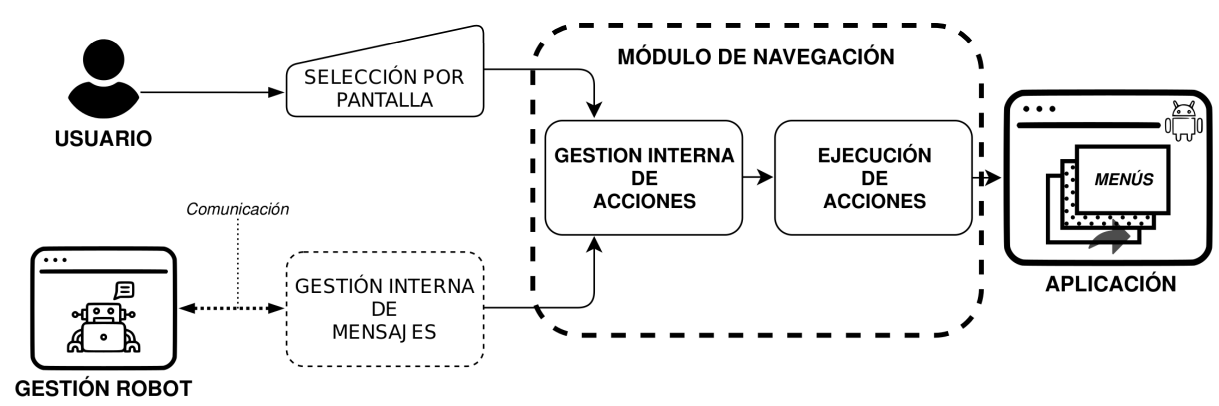

(c) Esquema de la estructura del módulo de navegación

Figura 3: Diagramas de los módulos de la aplicación

En la Fig. 3c se representa el esquema general de la estructura del módulo de navegación.

\subsubsection{Módulo reproductor de música}

Actividad que proporciona al usuario la experiencia de escuchar música o programas de podcasts. Se trata de una interfaz gráfica con las funcionalidades de pausar, reanudar, avanzar o retroceder en la lista de música seleccionada.

Finalmente, el módulo se apoya en una librería interna de listas de contenido detallados en la siguiente sección.

\subsection{BIBLIOTECA BASADA EN LAS PREFERENCIAS MUSICALES DE LAS PERSONAS MAYORES}

De cara a la construcción de una librería predeterminada de contenido musical y programas de podcasts, se ha realizado un estudio previo con el fin de atender las preferencias de las personas mayores. Analizando los resultados de los géneros musicales más votados, se han construido menús de selección e interfaces gráficas específicos para cada uno.

Los resultados obtenidos de las encuestas se encuentran representados en la Figura 5.

La creación de dichas listas se ha realizado directamente sobre la base de datos de Spotify. De esta manera la aplicación desarrollada accede remotamente a cualquiera de las listas de música creadas. 


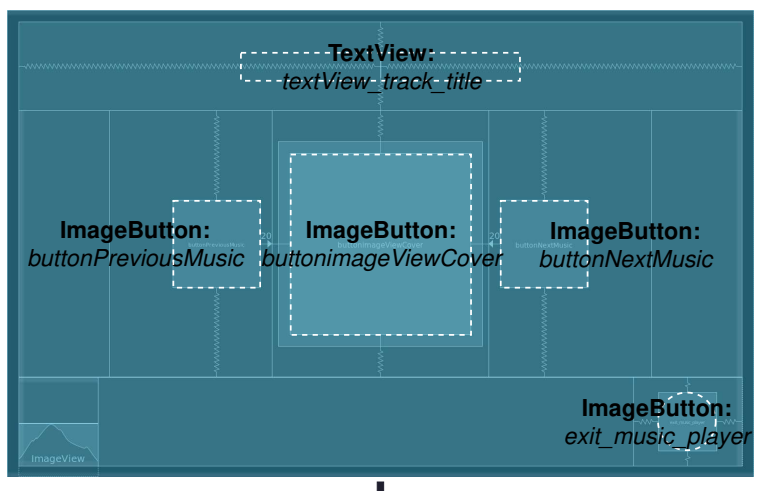

$\downarrow$

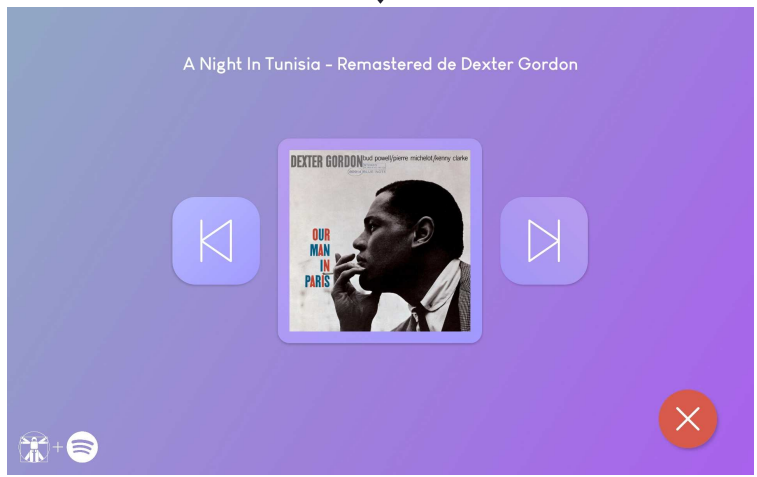

Figura 4: Diseño de la interfaz gráfica del módulo de reproducción

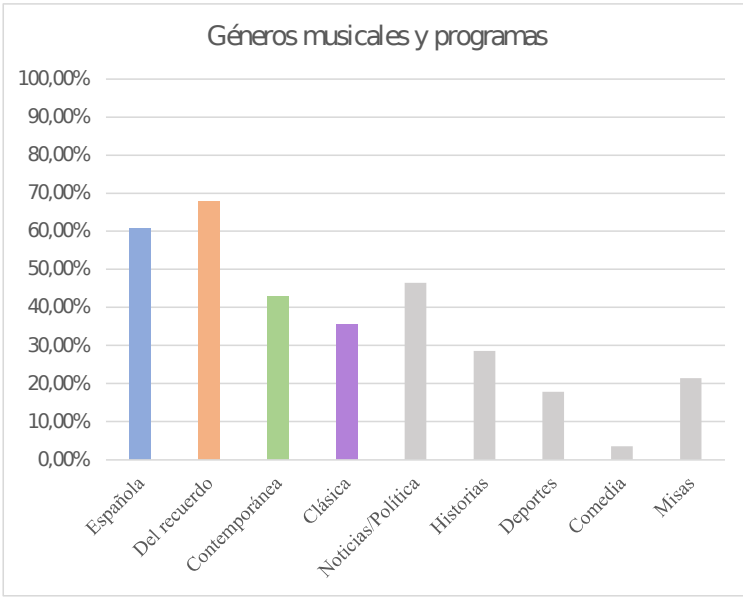

Figura 5: Gráfica de barras porcentual de contenido escuchado por las personas mayores encuestadas

\section{CONCLUSIONES}

En este artículo se ha realizado un repaso acerca de la robótica social e inclusión digital de la población mayor, haciendo especial mención a las aplicaciones accesibles que encontramos para usuarios mayores. Se han descrito las características principales que debería incorporar la aplicación propuesta y se ha presentado una aplicación, estructurada en cuatro módulos funcionales, que ofrece un servicio de reproducción de música vía strea- ming para el robot social Mini.

Referente al diseño de la aplicación desarrollada, se ha conseguido integrar el entorno de Android con módulos de comunicación con ROS. Estos módulos han permitido al programa usar el ASR y ETTS del robot Mini, cumpliendo el objetivo de desarrollar un aplicación más accesible.

Además, se ha conseguido construir una librería predeterminada de contenido musical basada en las encuestas realizadas a personas mayores. Esto ha permitido a la aplicación contar con un total de 20 listas de música y 21 programas de podcasts. Contenido razonable para el programa, con la posibilidad de mejorar o aumentar dichos contenidos en el futuro.

Finalmente, se concluye presentando la aplicación como base para encaminar el desarrollo de nuevas aplicaciones en el robot social Mini. La aplicación ha demostrado poder comunicarse sin problemas con el entorno del robot (ROS) llegando a mejorar aspectos tanto visuales y funcionales, como aspectos relacionados con accesibilidad y la experiencia de usuario.

\section{Agradecimientos}

La investigación desarrollada ha recibido financiación de dos proyectos: "Development of social robots to help seniors with cognitive impairment" (ROBSEN), financiado por el Ministerio de Economía y Competitividad; y "RoboCity2030IIICM", financiado por la Comunidad de Madrid y cofinanciado por los Fondos Estructurales de la Unión Europea.

\section{English summary}

\section{ACCESSIBLE MUSIC STREA- MING APPLICATION FOR SO- CIAL ROBOT}

\begin{abstract}
Social robotics continues to adapt to the changes taking place in the world and society today. The emergence of COVID-19 has led to a scenario of social distancing that has greatly affected at-risk populations such as the elderly.

However, the various interventions by social robotics have demonstrated their ability to combat a pandemic, offering services in hospitals, nursing homes and providing companionship to elderly people living alone. As a result, the idea of boosting the va-
\end{abstract}


lue of the social robot as a service platform has emerged.

For this reason, the present work proposes the design and implementation of a music and podcast playback application focused on the preferences of the elderly. Our development aims to promote accessibility and the use of multimedia content streaming services for the elderly. In parallel, the developed application aims to create new Android applications that support the social robots of the University Carlos III of Madrid and increase their competences in terms of services.

Keywords: Social robotics; Streaming services; Accessible applications; Music playback; Digital inclusion.

\section{Referencias}

[1] A. D. M. Africa, G. Ching, K. Go, R. Evidente y J. Uy, «A comprehensive study on application development software systems,» International Journal of Emerging Trends in Engineering Research, vol. 7, n. ${ }^{\circ} 8$, pp. 99-103, 2019.

[2] F. Alonso-Martín y M. A. Salichs, «Integration of a voice recognition system in a social robot,» Cybernetics and Systems, vol. 42, n. ${ }^{\circ} 4$, pp. 215-245, 2011.

[3] F. Alonso-Martin, A. Ramey y M. Á. Salichs, «Maggie : el robot traductor,» en 9 Workshop RoboCity2030-II, Breazeal 2003, 2011, pp. 57-73.

[4] M. Čaić, D. Mahr y G. OderkerkenSchröder, "Value of social robots in services: social cognition perspective,» Journal of Services Marketing, vol. 33, n. ${ }^{\circ} 4$, pp. 463-478, 2019.

[5] J. C. Cobo Romaní, «El concepto de tecnologías de la información. Benchmarking sobre las definiciones de las TIC en la sociedad del conocimiento,» vol. 14, 2009.

[6] P. Farhadi, «The Social Presence of Jibo,» Tesis doct., 2019, pp. 1-61.

[7] A. P. Henkel, M. Caić, M. Blaurock y M. Okan, «Robotic transformative service research: deploying social robots for consumer well-being during COVID-19 and beyond,» Journal of Service Management, vol. aheadof-p, n. ${ }^{\circ}$ ahead-of-print, 2020.
[8] M. B. Hoy, «Alexa, Siri, Cortana, and More: An Introduction to Voice Assistants,» Medical Reference Services Quarterly, vol. 37, n. ${ }^{\circ} 1$, pp. 81-88, 2018.

[9] K. Jacobson, V. Murali, E. Newett, B. Whitman y R. Yon, «Music Personalization at Spotify,» Association for Computing Machinery (ACM), 2016, pp. 373-373.

[10] «Let me Introduce Myself: I am Care-Obot 4, a Gentleman Robot,» en Mensch und Computer 2015 - Tagungsband, 2015, pp. 223-232.

[11] J. Liu y J. Yu, «Research on development of android applications,» en Proceedings - 2011 4 th International Conference on Intelligent Networks and Intelligent Systems, ICINIS 2011, 2011, pp. 69-72.

[12] M. Maroto-Gómez, Á. Castro-González, J. C. Castillo, M. Malfaz y M. A. Salichs, $\ll$ A bio-inspired motivational decision making system for social robots based on the perception of the user,» Sensors (Switzerland), vol. 18, n. ${ }^{\circ}$ 8, p. 2691, 2018.

[13] X. Martínez-Rolán y T. Piñero-Otero, «Tipología y funcionalidades de las aplicaciones móviles para mayores. A un tap del envejecimiento activo,» Ámbitos. Revista Internacional de Comunicación, n. ${ }^{\circ}$ 29, 2015.

[14] A. K. Pandey y R. Gelin, «A Mass-Produced Sociable Humanoid Robot: Pepper: the First Machine of Its Kind,» IEEE Robotics and Automation Magazine, vol. 25, n. ${ }^{\circ} 3$, pp. 40-48, 2018.

[15] A. J. Pérez Vidal, Á. Castro-González, F. Alonso Martín, J. C. Castillo y M. Á. Salichs, «Evolución de la robótica social y nuevas tendencias,» Universidade da Coruna, 2020, pp. 836-843

[16] J. Ramírez-Ortiz, D. Castro-Quintero, C. Lerma-Córdoba, F. Yela-Ceballos y F. Escobar-Córdoba, «Consequences of the COVID-19 pandemic in mental health associated with social isolation - Consecuencias de la pandemia COVID-19 en la salud mental asociadas al aislamiento social,» 2020

[17] A. Rebollo Ena, «SPOTIFY / NETFLIX Nuevos modelos de negocio,» Bit, n. ${ }^{\circ} 210$, pp. 41-43, 2018.

[18] E. Salichs, E. Fernández-Rodicio, J. C. Castillo, Á. Castro-González, M. Malfaz y M. Á. Salichs, «A social robot assisting in cognitive stimulation therapy,» en Lecture Notes in Computer Science (including subseries Lecture Notes in Artificial Intelligence and Lecture Notes in Bioinformatics), vol. 10978 LNAI, Springer Verlag, 2018, pp. 344-347. 
[19] M. A. Salichs, Á. Castro-González, E. Salichs, E. Fernández-Rodicio, M. MarotoGómez, J. J. Gamboa-Montero, S. MarquesVillarroya, J. C. Castillo, F. Alonso-Martín y M. Malfaz, «Mini: A New Social Robot for the Elderly,» International Journal of Social Robotics, vol. 12, n. ${ }^{\circ}$ 6, pp. 1231-1249, 2020.

[20] D. Skog, H. Wimelius y J. Sandberg, «Digital Service Platform Evolution: How Spotify Leveraged Boundary Resources to Become a Global Leader in Music Streaming,» en Proceedings of the 51st Hawaii International Conference on System Sciences, Hawaii International Conference on System Sciences, 2018.

[21] TvUS, Brecha Digital y e-inclusión de las personas mayores: grandes desafíos de la sociedad actual.

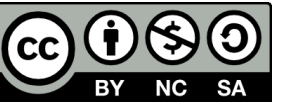

(C) 2021 by the authors. Submitted for possible open access publication under the terms and conditions of the Creative Commons Attribution CC BY-NC-SA 4.0 license (https://creativecommons.org/licenses/by-ncsa/4.0/deed.es). 